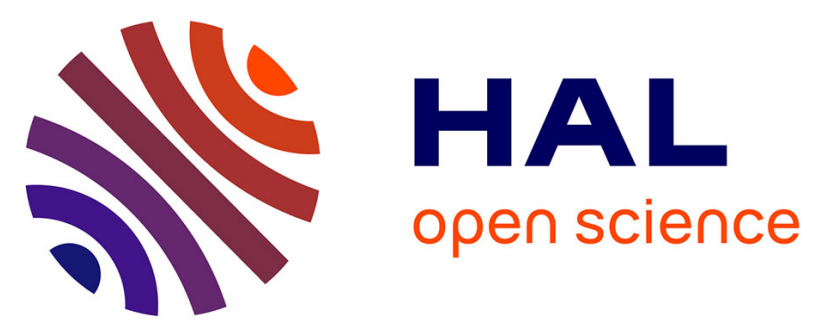

\title{
Development and characterization of layer-by-layer coated liposomes with poly(L-lysine) and poly(L-glutamic acid) to increase their resistance in biological media
}

Florence Hermal, Benoit Frisch, Alexandre Specht, Beatrice Heurtault, Line Bourel-Bonnet

\section{To cite this version:}

Florence Hermal, Benoit Frisch, Alexandre Specht, Beatrice Heurtault, Line Bourel-Bonnet. Development and characterization of layer-by-layer coated liposomes with poly(L-lysine) and poly(L-glutamic acid) to increase their resistance in biological media. International Journal of Pharmaceutics, 2020, 586, pp.119568. 10.1016/j.ijpharm.2020.119568 . hal-02991433

\section{HAL Id: hal-02991433 \\ https://hal.science/hal-02991433}

Submitted on 6 Nov 2020

HAL is a multi-disciplinary open access archive for the deposit and dissemination of scientific research documents, whether they are published or not. The documents may come from teaching and research institutions in France or abroad, or from public or private research centers.
L'archive ouverte pluridisciplinaire HAL, est destinée au dépôt et à la diffusion de documents scientifiques de niveau recherche, publiés ou non, émanant des établissements d'enseignement et de recherche français ou étrangers, des laboratoires publics ou privés. 


\section{Development and Characterization of Layer-by-Layer Coated Liposomes to Increase their Resistance in Biological Media}

Florence Hermala ${ }^{a}$ Benoît Frisch ${ }^{\mathrm{a}}$, Alexandre Specht ${ }^{\mathrm{b}}$, Line Bourel-Bonnet ${ }^{\mathrm{a}, 1,{ }^{*}}$ and Béatrice Heurtaulta,1, 5

a 3BIO Team, UMR 7199, Université de Strasbourg/CNRS, Faculté de Pharmacie, 74 route du Rhin, 67401 Illkirch Cedex, France

b CNM Team, UMR 7199, Université de Strasbourg/CNRS, Faculté de Pharmacie, 74 route du Rhin, 67401 Illkirch Cedex, France

$0 \quad{ }^{*}$ Corresponding authors

Email addresses: bheurtault@unistra.fr (B. Heurtault) and line.bourel@unistra.fr (L. Bourel-Bonnet) ${ }^{1}$ Equal contribution, co-last authors

\section{Abstract}

Multilayered coated liposomes were prepared using the layer-by-layer (LbL) technique in an effort to improve their stability in biological media. The formulation strategy was based on the alternate deposition of two biocompatible and biodegradable polyelectrolytes - poly(L-lysine) (PLL) and poly(L-glutamic acid) (PGA) - on negatively charged small unilamellar vesicles (SUVs). Some 0 parameters of the formulation process were optimized such as the polyelectrolyte concentration and

Abbreviations: 5(6)-CF, 5(6)-carboxyfluorescein; BSA, bovine serum albumin; Chol, cholesterol; DIEA, N,Ndiisopropylethylamine; DLS, dynamic light scattering; DMSO, dimethyl sulfoxide; EDC, 1-ethyl-3-(3dimethylaminopropyl)carbodiimide; FRET, Förster resonance energy transfer; HEPES, 4-(2-hydroxyethyl)-1piperazineethanesulfonic acid; IV, intravenous; LbL, layer-by-layer; Lp, liposomes; MLVs, multilamellar vesicles; NMR, nuclear magnetic resonance; OD, optical density; PC, phosphatidylcholine; PdI, polydispersity index; PES, poly(ether sulfone); PG, phosphatidylglycerol; PGA, poly(L-glutamic acid); PL, phospholipids; PLL, poly(L-lysine); RITC, rhodamine B isothiocyanate; SD, substitution degree; SDS, sodium dodecyl sulfate; sPLA, secretory phospholipase $A_{2}$; sulfo-NHS, Nhydroxysulfosuccinimide; SUVs, small unilamellar vesicles 
the purification procedure. This optimized procedure has allowed the development of very homogeneous formulations of liposomes coated with up to 6 layers of polymers (so-called layersomes). The coating was characterized by dynamic light scattering (DLS), zeta potential measurements and Förster resonance energy transfer (FRET) between two fluorescently labeled

5 polyelectrolytes. Studies on the stability of the formulations at $4^{\circ} \mathrm{C}$ in a buffered solution have shown that most structures are stable over 1 month without impacting their encapsulation capacity. In addition, fluorophore release experiments have demonstrated a better resistance of the layersomes in the presence of a non-ionic detergent (Triton $\left.{ }^{\mathrm{TM}} \mathrm{X}-100\right)$ as well as in the presence of phospholipase $\mathrm{A}_{2}$ and human plasma. In conclusion, a novel formulation strategy of multilayered coated liposomes has 0 been developed to increase the stability of conventional liposomes in biological environment.

Keywords: Layer-by-layer coating - liposomes - polyelectrolytes - poly(L-lysine) - poly(L-glutamic acid) - stability

\section{1. Introduction}

Conventional liposomes are promising drug delivery candidates because they are easy to prepare and industrially transposable, totally biocompatible and fully biodegradable.(Bozzuto and Molinari, 2015) Known as spherical vesicles formed of one or several concentric lipid bilayers 0 trapping one or more aqueous compartment(s), these nanoparticles are mainly constituted of phospholipids and cholesterol. When used in their formulation, the latter stabilizes the lipid membrane, modulates its fluidity and reduces its permeability.(Vemuri and Rhodes, 1995) Liposomes display versatile loading capacities: their aqueous core is fillable with hydrophilic compounds whereas their lipid membrane(s) can contain hydrophobic substances.(Lamichhane et al., 2018)

5 Liposomes were applied in medical imaging,(Lamichhane et al., 2018; Petersen et al., 2012) vaccination (Marasini et al., 2017; Tandrup Schmidt et al., 2016) and theranostics,(Muthu and Feng, 2013; Xing et al., 2016) and also exploited in the therapeutical field as drug carriers (amphotericin B 
in Ambisome $\mathrm{TM}^{\mathrm{TM}}$, anthracyclines in Daunoxome $\mathrm{TM}^{\mathrm{TM}}$ or yyocet $^{\mathrm{TM}}$...), to control pain (morphine in Depopur $^{\mathrm{TM}}$ ) and treat macular degeneration related to age (verteporfin in Visudyne ${ }^{\mathrm{TM}}$ ).(Bulbake et al., 0 2017)

Yet the major drawback of conventional liposomes is their low stability in biological media. Thus, in blood, especially after intravenous (IV) injection, a rapid clearance is observed due to a minute to hour-scale uptake by macrophages (Bozzuto and Molinari, 2015; Sercombe et al., 2015) and 5 a frequent destabilization, mainly by circulating high-density plasma lipoproteins.(Bozzuto and Molinari, 2015) To overcome stability problems, liposomes can be either covalently reticulated or coated by a polymeric shell to become significantly more stable, notably in the above-mentioned physiological conditions. The most common example is the single-agent PEGylated liposomal (Stealth ${ }^{\mathrm{TM}}$ ) doxorubicin used in the chemotherapeutical fights against Kaposi's sarcoma, ovarian i0 cancer, breast cancer and multiple myeloma. With the corresponding approved drugs, the overall circulation time of doxorubicin is firmly increased while its cardiotoxicity is dramatically limited.(Nag and Awasthi, 2013) In the gastro-intestinal environment, a rapid degradation due to harsh $\mathrm{pH}$ conditions, bile salts and pancreatic enzymes is commonly encountered.(He et al., 2019; Liu et al., 2019) As a consequence, there is a need of improving conventional liposomes stability and resistance.

The layer-by-layer (LbL) coating of surfaces was first proposed by Decher and coworkers and corresponds to the alternate adsorption of complementary multivalent species onto a planar or spherical substrate via either electrostatic interactions, hydrogen bonding or other secondary interactions.(Decher et al., 1992; Decher and Hong, 1991a, 1991b) LbL coating can be obtained by '0 alternate immersion, spray or spin droplet drying (Richardson et al., 2016, 2015) and can involve peptides and proteins, nucleic acids, polysaccharides and any other polymers, either natural or synthetic.(Gentile et al., 2015) It can be optimized by the fine-tuning of several parameters like i/ the nature of the support, ii/ the nature and the concentration of polymeric counterparts, iii/ the nature and the ionic strength of the solvent and iv/ the overall experimental conditions (duration of 
5 contact/incubation, temperature, pH, separation technics...).(Guzmán et al., 2017) When mastered, all these parameters permit a fine control of the LbL coating properties. LbL coating has many advantages like simple and cheap formulation steps, mild assembly conditions, a large variety of species usable on a broad diversity of supports and more importantly, enables a precise physicochemical control of the resulting coating.(Ai et al., 2003; Gentile et al., 2015; Hammond, 2012, 2011)

:0 Thus LbL coating is a simple, versatile and controllable way to modify and functionalize surfaces and objects.(Gentile et al., 2015; Keeney et al., 2015; Liu and Picart, 2016)

In that wake, liposomes can be alternatively coated with complementary polyelectrolytes according to the LbL approach to get nanostructures so-called 'layersomes'.(He et al., 2019; Nguyen et 5 al., 2016) In rare examples, oppositely charged polymers like poly(acrylic acid) and poly(allylamine hydrochloride), have been previously used to increase the stability of conventional liposomes in simulated biological media.(Jain et al., 2009, 2012) Yet as PAH is not biocompatible,(Picart et al., 2005) alternative polymers were also tried with success toward an IV utilization like the poly(LLysine)/poly(ethylene glycol)-poly(L-Aspartic acid) copolymer (Ramasamy et al., 2014) and poly(L$10 \quad$ Arginine)/Hyaluronic acid couples.(Deng et al., 2013)

Here the strategy is to develop and characterize LbL coated liposomes using an alternative and relevant pair of polymers - poly-(L-Lysine) (PLL) and poly-L-glutamic acid (PGA) - that has not yet been investigated for the preparation of layersomes. These two polyelectrolytes were chosen for their 5 unique unprecedented combination of characteristics that include biocompatibility,(Tryoen-Tóth et al., 2002) bioresistance to hydrolysis (Leguen et al., 2007), use at physiological pH (7.4) and the possibility of easily modifying them chemically for example by covalently crosslinking their side chains.(Gribova et al., 2013; Schneider et al., 2006). The results obtained with 2-4-6 layered layersomes are presented below. A meticulous tuning of some parameters of the formulation 10 procedure such as the polymers concentration and the method of purification was conducted. Indeed, the formulation procedure had to permit a stable double to multiple layers coating in a reasonable 
experimental time. Moreover, homogenous suspensions of small and monodispersed nanoparticles (with diameters inferiors to $200 \mathrm{~nm}$ ) were highly desirable to open the way to an IV administration.(Blanco et al., 2015), which is rarely achieved with multilayered liposomes. The stability of the nanoparticles' suspensions upon storage was studied parallelly. Besides, a FRET experiment demonstrated the co-localization of the polymers onto the liposomes. Finally, investigations were carried out to explore the stability of layersomes toward surfactant (Triton ${ }^{\mathrm{TM}} \mathrm{X}$ 100), enzyme (phospholipase $A_{2}$ ) and human plasma through the release of a fluorophore. Encouraging results were obtained and prove here that layersomes formulated with this new $0 \quad$ combination of polymers could truly be regarded as promising stabilized liposomes.

\section{Experimental procedures}

\subsection{Chemical reagents and equipment}

$64 \mathrm{kDa}$ Poly(-L glutamic acid) sodium salt (PGA), rhodamine B isothiocyanate mixed isomers (RITC), 1-ethyl-3-(3-dimethylaminopropyl)carbodiimide (EDC) hydrochloride, Nhydroxysulfosuccinimide sodium salt (Sulfo-NHS), hydrochloric acid (HCl), L-ascorbic acid crystalline, molybdic acid ammonium salt 81 - $83 \% \mathrm{MoO}_{3}$, polyethylene glycol octylphenyl ether (Triton ${ }^{\mathrm{TM}} \mathrm{X}-100$ ) 0 and sodium dodecyl sulfate (SDS) were purchased from Sigma-Aldrich (Saint Louis, USA). 21 kDa Poly(L-lysine) hydrobromide (PLL) was purchased from Alamanda Polymers Inc (Huntsville, USA). Perchloric acid $70 \%$ solution in water and potassium phosphate monobasic $\left(\mathrm{KH}_{2} \mathrm{PO}_{4}\right)$ were purchased from Fisher Scientific (Hampton, USA). Sodium chloride ( $\mathrm{NaCl})$, sodium hydroxide $(\mathrm{NaOH})$ and potassium phosphate dibasic $\left(\mathrm{K}_{2} \mathrm{HPO}_{4}\right)$ were purchased from VWR International (Radnor, USA).

5 5-aminofluorescein was purchased from Honeywell (Charlotte, USA). 5(6)-carboxyfluorescein (5(6)CF) was purchased from Eastman Chemical Company (Kingsport, USA). N,N-diisopropylethylamine (DIEA) was purchased from Alfa Aesar (Ward Hill, USA). Calcium chloride dihydrate $\left(\mathrm{CaCl}_{2} \cdot 2 \mathrm{H}_{2} \mathrm{O}\right)$ was obtained from Merck (Darmstadt, Germany). (4-(2-hydroxyethyl)-1-piperazineethanesulfonic acid) 
pufferan (HEPES) was purchased from Carl Roth (Karlsruhe, Germany). All other anhydrous solvents $0 \quad$ were commercially available and used without further purification.

${ }^{1} \mathrm{H}$ NMR spectra, which showed a shift referenced to the residual signal of non-fully deuterated NMR solvents, were recorded on a Bruker Advance DPX400 NMR spectrometer locked to the major signal of each NMR solvent. Samples were dissolved in either $\mathrm{D}_{2} \mathrm{O}$ or DMSO- $\mathrm{d}_{6}$ (Sigma-Aldrich) at 25 $5 \quad{ }^{\circ} \mathrm{C}$.

\subsection{Lipids}

Egg yolk L- $\alpha$-phosphatidylcholine (PC) and cholesterol (Chol; recrystallized in $\mathrm{MeOH}$ ) were 0 purchased from Sigma-Aldrich. Egg yolk L- $\alpha$-phosphatidyl-DL-glycerol (PG) was purchased from Avanti Polar Lipids (Alabaster, USA). Lipid purities exceeded 99 \%. Lipids were stored under argon atmosphere in a powder form or dissolved in $\mathrm{CHCl}_{3} / \mathrm{MeOH}(9 / 1 \mathrm{v} / \mathrm{v})$ at a concentration of $\approx 25$ $\mu \mathrm{moL} . \mathrm{mL}^{-1}$ at $-20^{\circ} \mathrm{C}$. Concentrations of phospholipid (PL) solutions were determined by phosphate titration.(Rouser et al., 1970)

\subsection{Water, dialysis and filtration}

Ultrapure water (conductivity $=18 \mathrm{~m} \Omega$ ) was obtained by an ELGA Labwater device from Veolia (Aubervilliers, France). Millex-GP $0.22 \mu \mathrm{m}$ poly(ethersulfone) (PES) sterile syringe filters were 0 purchased from Merck Millipore (Burlington, USA). Sephadex ${ }^{\circledR}$ G-75 GE Healthcare was purchased from Sigma-Aldrich. Spectra/Por ${ }^{\mathrm{TM}} 3$ dialysis membranes of $3.5 \mathrm{kDa}$ and 5 mL Float-a-Lyzer ${ }^{\circledR} 2$ dialysis devices of $100 \mathrm{kDa}$ were purchased from Spectrum Labs Inc. (Phoenix, USA). 10 mL 8003 Amicon $^{\mathrm{TM}}$ pressure concentrator and $25 \mathrm{~mm}$ diameter ultrafiltration discs of $100 \mathrm{kDa}$ (Ultracel regenerated cellulose) were purchased from Merck. 15 mL Vivaspin Turbo protein concentrators of $5100 \mathrm{kDa}$ (PES) were purchased from Sartorius (Göttingen, Germany). 


\subsection{Biological reagents}

Bovine serum albumin (BSA) was purchased from Sigma-Aldrich. Secretory phospholipase $\mathrm{A}_{2}$

i0 $\left(\mathrm{sPLA}_{2}\right)$ type III originating from bee venom was obtained from Bertin Bioreagent (Montigny-leBretonneux, France). Human plasma was purchased from the Établissement Français du Sang (La Plaine Saint-Denis, France).

\subsection{Chemical syntheses}

\subsubsection{Synthesis of PLL-rhodamine}

$42 \mathrm{mg}(0.2 \mathrm{mmol}$ of monomer) of $21 \mathrm{kDa}$ PLL were solubilized in $2 \mathrm{~mL}$ of anhydrous DMSO then $270 \mu \mathrm{L}$ ( $2 \mathrm{mmol}$ ) of DIEA were added to it. The neutralization mixture was placed under stirring at '0 room temperature for $15 \mathrm{~min}$. Then, $11 \mathrm{mg}(0.02 \mathrm{mmol})$ of rhodamine B isothiocyanate (RITC) were solubilized in $0.4 \mathrm{~mL}$ of anhydrous DMSO and added to the polyamine. The reaction was left overnight under stirring and argon atmosphere, protected from light and at room temperature. Afterwards, grafted PLL was precipitated in $20 \mathrm{~mL}$ isopropanol and consequently centrifuged at 10,000 g for 10 min. This washing process was repeated 5 times. Then, PLL-rhodamine was solubilized in $10 \mathrm{~mL}$

5 ultrapure water and dialyzed (Spectra/Por ${ }^{\mathrm{TM}} 3$ dialysis membrane $3.5 \mathrm{kDa}$ ) against $1.5 \mathrm{~L}$ of $150 \mathrm{mM}$ $\mathrm{NaCl}$ overnight and finally against ultrapure water until no significant fluorescence was detected in the dialysis medium. The product was then lyophilized, leading to $32 \mathrm{mg}$ of a purple solid (yield: 90 \%). The substitution degree of the rhodamine-labeled PLL was determined by absorbance at $560 \mathrm{~nm}$ in $0.1 \mathrm{M} \mathrm{pH} 8$ phosphate buffer using a standard curve(Mladenovska et al., 2007) and was found to be : 0 of $3 \%$.

\subsubsection{Synthesis of PGA-fluorescein}


$105 \mathrm{mg}$ ( $0.7 \mathrm{mmol}$ of monomer) of $64 \mathrm{kDa}$ PGA sodium salt were solubilized in $3 \mathrm{~mL}$ of $10 \mathrm{mM}$

HEPES pH 6.5. $29 \mathrm{mg}(0.15 \mathrm{mmol})$ of EDC and $3 \mathrm{mg}(0.015 \mathrm{mmol})$ of sulfo-NHS solubilized in 150 and $50 \mu \mathrm{L}$ of $10 \mathrm{mM}$ HEPES pH 6.5, respectively, were added to the PGA solution. The activation was left under stirring at room temperature for $20 \mathrm{~min}$. Then, $36 \mathrm{mg}(0.1 \mathrm{mmol})$ of 5-aminofluorescein were solubilized in $1.5 \mathrm{~mL}$ of anhydrous DMSO and added to the reaction. The reaction was left overnight under stirring and argon atmosphere, protected from light, and at room temperature. Afterwards, the 0 obtained grafted PGA was precipitated in $20 \mathrm{~mL}$ of isopropanol and consequently centrifuged at $10,000 \mathrm{~g}$ for $10 \mathrm{~min}$. This washing process was repeated 5 times. Then, PGA-fluorescein was solubilized in $10 \mathrm{~mL}$ of ultrapure water and dialyzed against $1.5 \mathrm{~L}$ of $150 \mathrm{mM} \mathrm{NaCl}$ overnight using a $3.5 \mathrm{kDa}$ dialysis membrane (Spectra/Por ${ }^{\mathrm{TM}}$ 3) and finally against ultrapure water until no significant fluorescence was detected in the dialysis medium. The product was then lyophilized, leading to $76 \mathrm{mg}$ of a yellow solid (yield: 72\%). ${ }^{1} \mathrm{H}$ NMR, $\mathrm{D}_{2} \mathrm{O}: \delta(\mathrm{ppm})=2.21-2.58\left(\mathrm{~m}, 4 \mathrm{H}, \mathrm{H}_{\beta}\right.$ and $\left.\mathrm{H}_{\gamma}\right), 4.31\left(\mathrm{~s}, 1 \mathrm{H}, \mathrm{H}_{\alpha}\right)$, 6.79-6.93 (m, 4H, $\mathrm{H}_{5}, \mathrm{H}_{6}, \mathrm{H}_{7}$ and $\left.\mathrm{H}_{8}\right), 7.30-7.38\left(\mathrm{~m}, 3 \mathrm{H}, \mathrm{H}_{3}, \mathrm{H}_{4}\right.$ and $\left.\mathrm{H}_{9}\right), 7.78\left(\mathrm{~s}, 1 \mathrm{H}, \mathrm{H}_{2}\right), 8.14\left(\mathrm{~s}, 1 \mathrm{H}, \mathrm{H}_{1}\right)$, (Scheme 2). ${ }^{1} \mathrm{H}$ NMR spectroscopy was used to assess the substitution degree (SD) of PGA, which was $0.6 \%$. The integration value of $\mathrm{PGA}_{\alpha}$ signal at $\delta=4.31 \mathrm{ppm}$ was compared to that of 5aminofluorescein $\mathrm{H}_{2}$ at $\delta=7.78 \mathrm{ppm}$ by the following equation (1):

$$
S D(\%)=\frac{H_{2} \text { Amino-fluorescein }}{H_{\alpha} \text { PGA }} \#(1)
$$

\subsubsection{Synthesis of PGA-sulfo-NHS}

$300 \mathrm{mg}$ (2.0 mmol of monomer) of $64 \mathrm{kDa}$ PGA sodium salt were dissolved in $20 \mathrm{~mL}$ of ultrapure water and dialyzed against $1.5 \mathrm{~L}$ of $0.1 \mathrm{M}$ aqueous $\mathrm{HCl}$ using a $3.5 \mathrm{kDa}$ dialysis membrane (Spectra/Por 3). $128 \mathrm{mg}$ (1.0 mmol) of a white solid were obtained after drying under vacuum for 24 h. Then, $30 \mathrm{mg}(0.2 \mathrm{mmol})$ of PGA-COOH were dissolved in $0.6 \mathrm{~mL}$ of anhydrous DMSO. $22 \mathrm{mg}$ of EDC 
$(0.1 \mathrm{mmol})$ and $23 \mathrm{mg}(0.1 \mathrm{mmol})$ of sulfo-NHS were dissolved in $1.2 \mathrm{~mL}$ of anhydrous DMSO and added to the PGA-COOH solution. The reaction was left overnight under stirring and argon at room 0 temperature. Then, the crude was washed with $80 \mathrm{~mL}$ of acetone to remove free EDC and sulfo-NHS and it was centrifuged for $10 \mathrm{~min}$ at 10,000 g. This washing process was repeated 4 times. The obtained product was dried under vacuum. $40 \mathrm{mg}$ of product were obtained and stored under argon atmosphere and protected from water at $-20^{\circ} \mathrm{C}$ (yield: $\left.>99 \%\right) .{ }^{1} \mathrm{H} N M R, \mathrm{D}_{2} \mathrm{O}: \delta(\mathrm{ppm})=8.00-8.76$ $(\mathrm{m}, \approx 1 \mathrm{H}, \mathrm{NH}), 4.43-4.58\left(\mathrm{~m}, 1 \mathrm{H}, \mathrm{H}_{\alpha}\right), 4.33-4.38\left(\mathrm{~m}, 1 \mathrm{H}, \mathrm{H}_{1}\right), 3.06\left(\mathrm{sd}, 2 \mathrm{H}, \mathrm{H}_{2}\right)$, (Fig. 7). ${ }^{1} \mathrm{H}$ NMR 5 spectroscopy was used to assess the substitution degree of PGA, which was $50 \%$. The integration value of the $\mathrm{H}_{\alpha}$ proton of PGA signal at $\delta=4.43-4.58$ ppm was compared to that of sulfo-NHS $\mathrm{H}_{2}$ proton at $\delta=3.06 \mathrm{ppm}$ by the following equation (2):

$$
S D(\%)=\frac{H_{2} \mathrm{NHS}}{H_{\alpha} \mathrm{PGA}} \#(2)
$$

\subsection{Preparation of liposomes}

Multilamellar vesicles (MLVs) were prepared by hydration of a lipid film as described previously.(Kakhi et al., 2015) Briefly, a chloroform/methanol solution 9/1 v/v) containing a mixture of PC, PG and Chol (54:13:33 molar ratios) was deposited in round-bottom Pyrex ${ }^{\circledR}$ tubes and 5 completely dried under vacuum for $45 \mathrm{~min}$. The resulting lipid film was hydrated with $1.5 \mathrm{~mL}$ of 0.22 $\mu \mathrm{m}$ filtered buffered aqueous solution (10 mM HEPES, $15 \mathrm{mM} \mathrm{NaCl}, \mathrm{pH} 7.4$ ) with or without 5(6)-CF at $200 \mathrm{mM}$ and subsequently vortexed for $10 \mathrm{~min}$. The prepared MLVs were sonicated using a stepped 1/8" microtip (Delta Labo, Avignon, France) and a Vibra Cell ultrasonicator (750 W, 20 kHz, Fisher Scientific) under argon atmosphere for $1 \mathrm{~h}$ and protected from light to obtain small unilamellar 0 vesicles (SUVs). The obtained SUVs were then centrifuged $10 \mathrm{~min}$ at 10,000 $\mathrm{g}$ to pellet and remove titanium residues originating from the sonication probe. For liposomal suspensions containing 5(6)CF (Lp-5(6)-CF), the free dye was eliminated by filtration on a $20 \mathrm{~mL}$ Sephadex ${ }^{\circledR}$ G75 size exclusion 
column preliminarily equilibrated with $100 \mathrm{~mL}$ of buffer (10 mM HEPES, $15 \mathrm{mM} \mathrm{NaCl}, \mathrm{pH}$ 7.4).

Filtered SUVs were stored at $4^{\circ} \mathrm{C}$ under argon atmosphere.

\subsection{Preparation of layersomes}

Layersomes were prepared according to a procedure previously published with minor modifications.(Ciobanu et al., 2007) In short, $3 \mathrm{~mL}$ of plain liposomal suspension at a concentration of $0 \quad 0.67 \mathrm{mM}$ of phospholipids were slowly dropped (30 drops. $\mathrm{min}^{-1}$ ) in a $3 \mathrm{~mL}$ PLL solution at [0.1 - 1.5 mg.mL $L^{-1}$ placed under stirring at $4^{\circ} \mathrm{C}$. After 20 min stirring, the polymer excess was removed either by dialysis against buffer (10 mM HEPES, 15 mM NaCl, pH 7.4, Float-a-Lyzer ${ }^{\circledR} 2$ dialysis devices 100 $\mathrm{kDa}$ ) or by filtration (Vivaspin Turbo protein concentrator $100 \mathrm{kDa}$ ). Once purified, the liposome-PLL (Lp-PLL) suspensions were slowly dropped in a PGA solution of the same volume at [0.1 - 6 mg.mL-1 $]$

5 placed under stirring at $4^{\circ} \mathrm{C}$. For the formulation of reticulated layersomes, $50 \%$ grafted PGA-sulfoNHS was used at a concentration of $0.16 \mathrm{mg} \cdot \mathrm{mL}^{-1}$ instead of $64 \mathrm{kDa}$ PGA. After 20 min stirring, the suspensions were either filtrated or dialyzed to remove free polymer. To obtain up to 6-layer coated liposomes, this procedure was repeated. To avoid the formation of particle aggregates, the phospholipids concentration of layersomal suspensions was gradually reduced according to the $0 \quad$ number of polyelectrolyte layers added onto liposomes (Table 1).

\section{Table 1}

Phospholipids concentration of the layersome formulations adjusted for the addition of a supplementary polyelectrolyte layer. 


\begin{tabular}{ll}
\hline Formulation & Phospholipids concentration (mM) \\
\hline Lp-PLL-PGA & 0.40 \\
Lp-PLL-PGA-PLL & 0.30 \\
Lp-(PLL-PGA) & \\
Lp-(PLL-PGA) & 0.20 \\
\hline
\end{tabular}

To evaluate the incidence of polymer concentration, plain Lp-PLL were formulated with increasing concentrations of PLL (0.1 to $1.5 \mathrm{mg} \cdot \mathrm{mL}^{-1}$ ). For each Lp-PLL suspension prepared, 2 PGA concentrations (twice and fourth the PLL concentration in question) were investigated for the i0 formulation of 2-layer coated liposomes (Lp-PLL-PGA).

To optimize the polymer elimination procedure, plain Lp-PLL suspensions were prepared with $0.1 \mathrm{mg} \cdot \mathrm{mL}^{-1}$ of a PLL solution and the excess of polyelectrolyte was removed by filtration using either a pressure concentrator (Amicon ${ }^{\mathrm{TM}}$ pressure concentrator) or a centrifugal concentrator (Vivaspin 5 Turbo protein concentrator) with $100 \mathrm{kDa}$ cut-offs. For this purpose, $6 \mathrm{~mL}$ of Lp-PLL were placed in the concentrators pre-rinsed with ultrapure water and were 3 times concentrated at $4^{\circ} \mathrm{C}$. A pressure of 1.0 bar was applied for filtration with the pressure concentrator and the concentrated suspensions were obtained in a few minutes. The Lp-PLL filtered with the centrifugal concentrator were centrifuged at $100 \mathrm{~g}$ for $30 \mathrm{~min}$. PL losses were determined by phosphate titration.(Rouser et al., (0 1970)

For the FRET experiment, plain liposomes were coated with $3 \%$ grafted PLL-rhodamine and $0.6 \%$ grafted PGA-fluorescein at a concentration of $0.1 \mathrm{mg} \cdot \mathrm{mL}^{-1}$. As a control, plain liposomes were coated with non-fluorescent PLL and $0.6 \%$ grafted PGA-fluorescein at a concentration of 0.1 mg.mL $\mathrm{mL}^{-1}$. 5 Polymers excesses were removed by dialysis to avoid a destabilization of the samples (HEPES 10 mM, $\mathrm{NaCl} 15$ mM, pH 7.4, Float-a-Lyzer ${ }^{\star}$ G2 dialysis devices $100 \mathrm{kDa}$ ). 
All suspensions were stored at $4^{\circ} \mathrm{C}$ under argon atmosphere.

\section{2.8. Physico-chemical characterization of liposomes and layersomes}

\subsubsection{Inorganic phosphate assay}

The phosphate content of the lipid solutions was determined by phosphate titration. (Rouser et al., 5 1970) Briefly, 25 and $50 \mu \mathrm{l}$ of $1 \mu \mathrm{mol} . \mathrm{ml}-1 \mathrm{PC}$ and PG solutions were deposited in triplicate in test tubes and heated for $2 \mathrm{~h}$ at $110^{\circ} \mathrm{C}$ to evaporate the organic solvents. Once the solvents had evaporated, $300 \mu \mathrm{l}$ of $70 \%$ perchloric acid was added to each tube. The samples were vortexed briefly and heated for 20 minutes at $190^{\circ} \mathrm{C}$. Then $1 \mathrm{ml}$ of ultrapure water and $500 \mu \mathrm{l}$ of solutions of $1,25 \%$ molybdic acid and $5 \%$ ascorbic acid were added to each tube. The samples were briefly mixed by vortexing and heated in a water bath at $100^{\circ} \mathrm{C}$ for 5 minutes. To stop the reaction, the tubes were transferred to a cold-water bath for 5 minutes. Finally, the samples were briefly stirred in the vortex and their optical density was determined at $820 \mathrm{~nm}$ using a microplate reader (SAFAS SP2000, Xenius 5801). Their phosphate content was determined from a calibration curve prepared with monobasic potassium phosphate.

\subsubsection{Phosphatidylcholine assay}

The PC content of formulated liposomes and layersomes was determined using an enzymatic assay with the LabAssay ${ }^{\mathrm{TM}}$ Phospholipid kit (Wako Pure Chemical industries) by colorimetric measurement with a SAFAS SP2000 spectrophotometer (Xenius 5801, SAFAS) as described previously.(Jacoberger-Foissac et al., 2019) Briefly, 1-2 $\mu \mathrm{L}$ of suspensions were incubated with $200 \mu \mathrm{L}$ of the enzymatic reagent in triplicates in a 96-well plate. The reagent contains phospholipase $\mathrm{C}$ that releases the choline, which is then oxidized by the choline oxidase. The reaction produces hydrogen 
peroxide needed by the peroxidase to convert a chromogen into a blue product. After $10 \mathrm{~min}$ at $37^{\circ} \mathrm{C}$, 15 absorbance was measured at $\lambda=595 \mathrm{~nm}$ using a microplate reader. PC content in each sample was determined from a calibration curve prepared with purified choline chloride.

\subsubsection{Particle size measurements}

The average diameter of formulated liposomes and layersomes was measured by dynamic light scattering (DLS) using a Zetasizer Nano-ZS (Malvern Panalytical, Malvern, UK) with the following specifications: material refractive index: 1.43; buffer refractive index: 1.33 ; viscosity: $0.9 \mathrm{cP}$; scattering angle: $90^{\circ}$; temperature: $25^{\circ} \mathrm{C}$. Liposomal and layersomal suspensions were diluted at a phospholipid concentration of $0.1 \mathrm{mM}$ in $10 \mathrm{mM}$ HEPES, $15 \mathrm{mM} \mathrm{NaCl}$ buffer, pH 7.4. Data were 5 analyzed using the multiple narrow mode included in the Malvern software. Particle size is expressed in intensity. Formulations are considered monodisperse when the polydispersity index (PdI) is $<0.3$.

\subsubsection{Zeta potential measurements}

Zeta potential of liposomes and layersomes was determined using a Zetasizer Nano-ZS. Samples were diluted at a phospholipid concentration of $0.2 \mathrm{mM}$ in $10 \mathrm{mM}$ HEPES, $15 \mathrm{mM}$ NaCl buffer, pH 7.4 and the zeta potential was calculated from the electrophoretic mobility based on the Smoluchowski approximation.

\section{$5 \quad$ 2.8.5. FRET analysis}

To confirm the LbL coating of liposomes by FRET, $500 \mu \mathrm{L}$ of fluorescent layersomes at a concentration of $0.03 \mathrm{mM}$ of phospholipids were inserted in a $1 \mathrm{~mL}$ Suprasil quartz cell (flx cell qz F semi-micro, SAFAS, Monaco). Emission spectra of fluorescein and rhodamine B were respectively 0 recorded from 500 to $700 \mathrm{~nm}$ and from 560 to $700 \mathrm{~nm}$ by exciting samples at the emission 
wavelength of fluorescein $\left(\lambda_{\mathrm{ex}}=488 \mathrm{~nm}\right)$ and rhodamine $B\left(\lambda_{\mathrm{ex}}=518 \mathrm{~nm}\right)$ and with the following settings: emission and excitation bandwidth: $10 \mathrm{~nm}$; step: $2 \mathrm{~nm}$; voltage: $500 \mathrm{~V}$. Baselines were recorded with buffer (10 mM HEPES, $15 \mathrm{mM} \mathrm{NaCl,} \mathrm{pH} \mathrm{7.4)} \mathrm{from} 500$ to $700 \mathrm{~nm}$ and from 560 to 700 $\mathrm{nm}$ under the same conditions as for samples. Emission spectra were recorded using a SAFAS SP2000 5 spectrometer and were instrumentally corrected by the correction algorithm displayed in the SAFAS software (SP2000 version 7, SAFAS).

\subsubsection{5(6)-CF encapsulation}

In order to evaluate their encapsulation stability, all layersome suspensions were prepared with liposomes containing 5(6)-carboxyfluorescein. In all cases, fluorescence measurements were performed in a $1 \mathrm{~mL}$ Suprasil quartz cell at constant voltage (410 V) using a SAFAS SP2000 spectrometer with the following specifications: $\lambda_{\mathrm{ex}}=488 \mathrm{~nm} ; \lambda_{\mathrm{em}}=518 \mathrm{~nm}$; bandwidth: $10 \mathrm{~nm}$. As described previously,(Ciobanu et al., 2007) 5(6)-CF entrapment efficiency was determined by 5 measuring the fluorescent signal of 5(6)-CF while embed into the liposomes/layersomes and once released from the particles using the following ratio (3):

$$
R=\frac{F_{\max }-F_{0}}{F_{\max }} \#(3)
$$

in which $F_{0}$ represents the initial fluorescence of the liposome suspensions and $F_{\max }$ the fluorescence 0 of released 5(6)-CF. For release of entrapped 5(6)-CF, $10 \mu \mathrm{L}$ of Triton ${ }^{\mathrm{TM}} \mathrm{X}-100$ were added to $600 \mu \mathrm{L}$ of liposomes/layersomes at a concentration of $0.006 \mathrm{mM}$ of phospholipids.

\subsection{Stability evaluation of layersomes}


In order to evaluate their stability, all layersome suspensions were prepared with liposomes containing 5(6)-carboxyfluorescein and in all cases, fluorescence measurements were performed following the same specifications as described in 2.8.5. To release all 5(6)-CF entrapped, $10 \mu \mathrm{L}$ of 100 $\%$ Triton $^{\mathrm{TM}} \mathrm{X}-100$ were added to the suspensions.

\subsubsection{With increasing concentrations of $\operatorname{Triton}^{\mathrm{TM}} \mathrm{X}-100$}

Bare liposomes as well as 2-, 4- and 6-layer coated layersomes were evaluated in the presence of increasing concentrations of Triton $^{\mathrm{TM}} \mathrm{X}-100$. For this purpose, $600 \mu \mathrm{L}$ of liposomal and layersomal suspensions were prepared at a concentration of $0.006 \mathrm{mM}$ of phospholipids and $10 \mu \mathrm{L}$ of an aqueous solution of Triton ${ }^{\mathrm{TM}} \mathrm{X}-100$ at $[0.075-0.9 \%](\mathrm{p} / \mathrm{v})$ were added to it. Fluorescence measurements of the suspensions were performed before and after the addition of the detergent.

\subsubsection{With phospholipase $A_{2}$}

Bare liposomes as well as 2-, 4- and 6-layer coated layersomes were evaluated in the presence of a phospholipase $\mathrm{A}_{2}$ (sPLA 2 type III, Bertin Bioreagent). For this purpose, $1 \mathrm{~mL}$ of liposomal and layersomal suspensions were prepared at a concentration of $0.006 \mathrm{mM}$ of phospholipids and $1 \mu \mathrm{L}$ of enzyme $(0.021 \mathrm{U} / \mathrm{mL})$ was added at $\mathrm{t}=0$ and $\mathrm{t}=15 \mathrm{~min}$ to the $1 \mathrm{~mL}$ of suspension. Then, the mixture was placed in a water bath set at $25^{\circ} \mathrm{C}$ for $75 \mathrm{~min}$. Fluorescence measurements of the suspensions 5 were measured before and at regular time intervals after the addition of the enzyme.

\subsubsection{With human plasma}

Bare liposomes as well as 2- and 4-layer coated layersomes were evaluated in the presence of

:0 human plasma. For this purpose, $267 \mu \mathrm{L}$ of liposomal and layersomal suspensions at a concentration of $0.05 \mathrm{mM}$ of phospholipids were diluted in $533 \mu \mathrm{L}$ of human plasma and were incubated for $2 \mathrm{~h}$ at 
$37^{\circ} \mathrm{C}$. Fluorescence measurements of the suspensions were measured before and after the addition of human plasma at regular time intervals with $90 \mu \mathrm{L}$ of sample diluted in $510 \mu \mathrm{L}$ of buffer $(10 \mathrm{mM}$ HEPES, $15 \mathrm{mM} \mathrm{NaCl}$, pH 7.4), to which $60 \mu \mathrm{L}$ of $20 \%$ SDS were added to denature plasmatic BSA.

\section{Results and discussion}

\subsection{Formulation of layersomes}

As starting material, SUVs composed of PC, PG and Chol (54:13:33 molar ratio) were prepared by a solvent evaporation process, followed by hydration of the obtained lipid films with HEPES in the presence of $\mathrm{NaCl}$ at $\mathrm{pH} 7.4$ and a consecutive in situ sonication step. They displayed an average diameter of $57 \pm 9 \mathrm{~nm}$ and a zeta potential of $-50 \pm 10 \mathrm{mV}$. The next step consisted in the alternate deposition of oppositely charged polyelectrolytes. First and as a polycation, poly(L-lysine) (PLL, 21

$\mathrm{kDa}$ ) was used as a bromhydrate salt. Second and as a polyanion, poly(L-glutamic acid) (PGA, $64 \mathrm{kDa}$ ) was employed as a carboxylate sodium salt (Fig. 1). Both species were selected for their biocompatibility and slow biodegradability. Moreover, and thanks to their side chains chemical reactivity, the two polymers could be chemically modified, either to be tracked, or to be cross-linked so as to convey a possible stability and/or a reinforced coating network of the layersomes.

The overall layersome preparation procedure required several steps. In brief, a diluted liposomal suspension was added dropwise to a PLL aqueous solution to get a Lp-PLL suspension via an anion/cation - here phosphate/ammonium - electrostatic interaction owing to properly chosen $\mathrm{pH}$ buffered conditions. Second, the Lp-PLL suspension was dialyzed for $24 \mathrm{~h}$ to remove the non15 wrapping/not adsorbed polymer. Third, the Lp-PLL suspension was transferred to a syringe and added dropwise to a PGA aqueous solution to get a Lp-PLL-PGA suspension via another anion/cation here carboxylate/ammonium - electrostatic interaction. The final Lp-PLL-PGA suspension was dialyzed for another $24 \mathrm{~h}$ to remove the non-wrapping/not adsorbed polymer and to reach the 
expected doubly coated suspension. Those operations could be repeated during several cycles to get

0 an even number of coatings $(2,4,6$ layers...) so as to obtain suspensions of layersomes of growing thickness (Fig.2).

As a proof of concept, two layer-coated layersomes were formulated and characterized by dynamic light scattering as shown in Fig. 3. Each addition of a polymer layer was associated with an 5 increase in size. Precisely, naked liposomes (Lp) of $57 \pm 9 \mathrm{~nm}$ grew to one-layer PLL-liposomes (LpPLL) of $71 \pm 11 \mathrm{~nm}$, then to double-layer liposomes (Lp-PLL-PGA) of $99 \pm 30 \mathrm{~nm}$, while every PdI remained under 0.3. A logical inversion of the overall charge was also observed. Precisely, the starting zeta potential (Lp: $-50 \pm 10 \mathrm{mV}$ ) turned positive (Lp-PLL: $+43 \pm 10 \mathrm{mV}$ ) and turned again negative (Lp-PLL-PGA: - $45 \pm 6 \mathrm{mV}$ ) along the alternate layers, first polycationic then polyanionic. As

0 experiments were repeated $(n=5)$ and similar results were obtained, a reproducible LbL coating was confidently suggested.

\subsection{Tuning and optimization of layersomes formulation}

\subsubsection{Optimization of the PLL and PGA concentrations}

To identify the most stable and homogeneous formulation, $21 \mathrm{kDa}$ PLL concentrations ranging from 0.1 to $1.5 \mathrm{mg} \cdot \mathrm{mL}^{-1}$ were investigated. Subsequently, two $64 \mathrm{kDa}$ PGA concentrations were employed (2 equiv. and 4 equiv., so from 0.2 and 0.4 mg.mL $\mathrm{m}^{-1}$ to 3.0 and $6.0 \mathrm{mg} \cdot \mathrm{mL}^{-1}$ ). Indeed, PGA

0 concentrations were always superior to those of PLL for dilution reasons, either to keep the same number of charges or to double it. A fine examination of size (mean diameter, PdI and width) and zeta potential results permitted to select the best experimental conditions.

For all PLL and PGA concentrations explored, average diameters close to $100 \mathrm{~nm}$ were obtained. 5 Studying the influence of PLL on the surface charge, whatever its concentration, similar zeta potential 
values $(+40 \pm 7 \mathrm{mV})$ were measured. The same trend was observed for PGA with zeta potential values of the order of - $40 \pm 6 \mathrm{mV}$ whatever the concentrations evaluated (Figs. S1 and S2). However, at concentrations of 0.1 mg.mL-1 PLL and 0.2/0.4 mg.mL ${ }^{-1}$, PGA, suspensions were more narrowly distributed in size and monodisperse than with superior concentrations. Consequently, these .0 concentrations were kept for the rest of the study (Fig. 4 A and B).

\subsubsection{Separation optimization}

To remove non-wrapped/not adsorbed free polymers, a dialysis filtration was initially performed. This operation eliminated excess of polyelectrolytes without impacting the physicochemical parameters of the layersomes. Nevertheless, dialysis required a considerable time as long as 24 hours and each addition of a bilayer lasted 2 days, consequently. Thus, there was a striking need for optimization of the separation/purification procedure.

Two other faster filtration techniques were therefore explored, which are ultrafiltration by centrifugation and ultrafiltration by pressure. The evaluation criteria were the size, homogeneity and zeta potential of 2-layer layersomes. Only ultrafiltration by centrifugation $\left(100 \mathrm{~g}\right.$ at $4^{\circ} \mathrm{C}$ with a 100 $\mathrm{kDa}$ filtration membrane) allowed us to quickly eliminate the polymeric species in 30 minutes without impacting the physico-chemical parameters of the layersomes. On the other hand, this technique had

5 the advantage of concomitantly concentrating the samples. This is why this filtration method was chosen for the rest of the present work.

\subsection{Demonstration of the LbL coating of liposomes - PGA/PLL proximity by FRET}


transfer (FRET) experiment was carried out. It required the synthesis of two FRET-compatible fluorescently labeled polyelectrolytes of the type PLL-rhodamine B and PGA-fluorescein. In this couple, fluorescein is the FRET donor $\left(\lambda_{\mathrm{ex}}=488 \mathrm{~nm}, \lambda_{\mathrm{em}}=518 \mathrm{~nm}\right)$ and rhodamine B is the FRET acceptor $\left(\lambda_{\mathrm{ex}}=543 \mathrm{~nm}, \lambda_{\mathrm{em}}=580 \mathrm{~nm}\right)$.(Sapsford et al., 2006; Sun et al., 2013)

\subsubsection{Synthesis of the fluorescently labeled polymers}

First, PLL was treated with 10 equiv. of N,N-diisopropyethylamine (DIEA) solubilized in DMSO to neutralize the ammonium. The free amine was then coupled to 0.1 equiv. of rhodamine B isothiocyanate (RITC) pre-solubilized in DMSO and the reaction was left under argon atmosphere at room temperature for $24 \mathrm{~h}$ (Scheme 1). The expected polymer (PLL-rhodamine, designated PLL*) was obtained in a good isolated yield of 92\%. A grafting percentage of $3 \%$ was quantified by UV '5 spectrophotometry.

In parallel, PGA was treated by 0.3 equiv. of 1-ethyl-3-(3-dimethylaminopropyl)carbodiimide (EDC), 0.03 equiv. of $\mathrm{N}$-hydroxysulfosuccinimide (sulfo-NHS) at pH 6.5 to get an extemporaneously activated carboxylic ester that was immediately coupled to 0.2 equiv. of 5 -aminofluorescein :0 previously solubilized in DMSO. The coupling was left over $24 \mathrm{~h}$ at room temperature under argon atmosphere to get the expected polymer (PGA-fluorescein, designated PGA*) with a good isolated yield of $72 \%$ (Scheme 2). A grafting percentage of $0.6 \%$ was determined via ${ }^{1} \mathrm{H}$ NMR assignment and integration of characteristic peaks (Equation 1, see 2.5.2.)

In conclusion, two fluorescently labeled polymers were successfully obtained. Whenever trying to increase the grafting, either less soluble polymers were obtained, or bad coating properties and undesired precipitations of the corresponding suspensions were observed.

\subsubsection{FRET experiment}


It was postulated that if the partially fluorescently labeled polyelectrolytes used as replacements for the usual PLL and PGA were in close proximity $(<10 \AA ̊)$ and correctly oriented, a Förster resonance energy transfer (FRET) might be possible. Two suspensions were then formulated: a negative control Lp-PLL-PGA* (where PGA-fluorescein replaced PGA) and Lp-PLL*-PGA* (where PLL-rhodamine and 5 PGA-fluorescein replaced PLL and PGA respectively). The fluorescence intensity measurements after excitation at $\lambda_{\mathrm{ex}} 488 \mathrm{~nm}$ gave the following curves (Fig. 5). Though weak, probably for grafting reasons $(0,6 \%$ and $3 \%)$, a FRET signal could clearly be detected at $580 \mathrm{~nm}$ in the Lp-PLL*-PGA* suspension and confirmed the close proximity of the polymers and therefore, undoubtedly demonstrated the LbL coating of so-called layersomes.

\subsection{Formulation and characterization of more complex layersomes}

In the case of a surface coating, the thickness of the polymer layers is an important parameter that can influence the strength of the coated material and its degradation kinetics.(Poon et al., 2011) 15 Therefore, larger polyelectrolyte multilayer coated layersomes were developed and characterized. These further layersomes contained 5(6)-carboxyfluorescein (5(6)-CF) encapsulated in the aqueous core of the liposomes (Lp-5(6)-CF). This hydrophilic fluorophore is classically used for the evaluation of liposome membrane permeability.(Weinstein et al., 2018)

For the formulation of larger layersomes, Lp-5(6)-CF with a 5(6)-CF concentration of $200 \mathrm{mM}$ were prepared and coated with up to 6 layers of PLL and PGA. As expected, an increase of the average diameter and a logical inversion of the zeta potential values were clearly observed at each step. Precisely, starting liposomes (Lp-5(6)-CF) were of $84 \mathrm{~nm}$ and increased regularly to reach 114, 111, 170, 203, 260 and $323 \mathrm{~nm}$ with 1, 2, 3, 4, 5 and 6 layers respectively. Starting Lp were negatively

5 charged $(-46 \pm 8 \mathrm{mV})$ and along the growing layers, the layersomes' charge alternated to display +37 , $-43,+32,-38,+26$ and $-35 \mathrm{mV} \pm 8 \mathrm{mV}$ with $1,2,3,4,5$ and 6 layers respectively. The formulations 
were monodispersed, as shown by the polydispersity indices (PdI) always inferior to 0.3 (Fig. 6) and were stable at physiological $\mathrm{pH}(7.4)$ over a month when stored at $4^{\circ} \mathrm{C}$.

On the other hand, 2-layer coated and reticulated layersomes were developed. These were obtained by formulating layersomes with PLL and a preliminary ester-activated PGA-sulfo-NHS (Fig. 7) with a grafting percentage of $50 \%$, which was determined via ${ }^{1} \mathrm{H}$ NMR assignment and integration of characteristic peaks (Equation 2, see 2.5.2.) That strategy leaded to the expected suspensions with similar DLS and zeta potential characteristics as classical 2-layer coated layersomes (Fig. S3).

Moreover, the 5(6)-CF encapsulation stabilities of the multilayered and reticulated constructs were evaluated and compared. To do so, fluorescence measurements were performed at constant voltage before and after their destruction as described previously.(Ciobanu et al., 2007) The release of 5(6)-CF is expressed as a ratio $\mathrm{R}$ (Equation 3 , see 2.8.5.). The higher the calculated $\mathrm{R}$ values, the 0 greater the amount of 5(6)-CF encapsulated. All layersomal formulations displayed similar released ratios $\mathrm{R}$ of 5(6)-CF over 1 month with values between 0.8 and 0.9 as those of naked liposomes, meaning that the coating does not influence their entrapment capacity (Table S1).

In conclusion, it was possible to formulate up to 6-layer and reticulated stable layersomes in a 5 reproducible way and with the same encapsulation properties regardless of the number of layers and the crosslinking.

\subsection{Stability evaluation of layersomes}

Once characterized, layersomes encapsulating 5(6)-CF were subjected to an evaluation of their stability in the presence of destabilizing elements. This stability study was carried out by evaluating the release of 5(6)-CF. For that, bi-, tetra-, hexa-layer layersomes and bilayer reticulated layersomes were studied. To begin with, the resistance of the layersomes was examined in the presence of a 
detergent, Triton ${ }^{\mathrm{TM}} \mathrm{X}-100$. Then, their resistance was studied in the presence of a phospholipase encountered in the digestive system ( $\mathrm{PPLA}_{2}$ ), and finally in the presence of human plasma (Fig. 8A, B and C).

\subsubsection{Stability evaluation in the presence of increasing concentrations of Triton ${ }^{\mathrm{TM}} \mathrm{X}-100$}

Layersomes were exposed to growing concentrations of non-ionic Triton ${ }^{\mathrm{TM}} \mathrm{X}-100$. This synthetic surfactant was chosen for its neutrality, its critical micellar concentration as low as 0.24-0.27 mM and its excellent ability to permeabilize membranes. Thus, it is a drastic non-natural detergent. In the following experiments, a progressive 5(6)-CF release was quickly observed for every formulation, indicating a clear destabilization in the presence of high surfactant percentage $(0.6 \mathrm{~m} / \mathrm{v}$ and over).

5 Nevertheless, at very low surfactant percentages ( $0.45 \mathrm{~m} / \mathrm{v}$ and lower), a difference between 6-layer liposomes and other formulations was obvious. The leak of 5(6)-CF was clearly less important (Fig. 8A). Thus, hexa-layer layersomes were encouragingly stable layersomes whereas reticulation did not improve the stability against deleterious Triton $^{\mathrm{TM}} \mathrm{X}-100$.

\subsubsection{Stability toward a phospholipase $A_{2}$}

According to the same principle, the stability of the layersomes was assessed in the presence of a phospholipase $\mathrm{A}_{2}\left(\mathrm{sPLA}_{2}\right)$, a hydrolase found in biological media, especially in the intestine. This enzyme specifically hydrolyzes the C2-ester function of glycerophospholipids and in its presence,

i5 conventional liposomes are rapidly destabilized.(Zhu et al., 2011) Here (Fig. 8B), most of the formulations of layersomes were poorly stable, except the formulation of hexa-layer layersomes that was a little more stable, confirming the protecting role of a sufficient polymeric thickness (at least 6 layers) around liposomes. 
Finally, the stability of bilayered +/- reticulated and tetralayered layersomes was evaluated in the presence of human plasma. It should be noted that considering its size superior than $200 \mathrm{~nm}$, the formulation of hexalayered layersomes was not subjected to this evaluation. From the Fig. 8C, all the '5 layered formulations released less 5(6)-CF than the uncoated liposomes over the entire duration of the study. Here, the cross-linked layersomes were the least resistant formulations. The most resistant were clearly the 4-layer layersomes.

To sum up, the stability of the layersomes evaluated by the release of 5(6)-CF has interestingly :0 shown improved resistance, especially for the 6-layer layersomes in the presence of Triton ${ }^{\mathrm{TM}} \mathrm{X}-100$ and phospholipase $\mathrm{A}_{2}$, and for 4-layer layersomes in the presence of human plasma.

\section{Conclusion}

In this study and for the first time, a LbL coating strategy using the biocompatible and biodegradable couple PLL (21 kDa) and PGA (64 kDa) was elaborated and optimized for the development of more stable liposomes in biological conditions. Using this assembly procedure, stable and monodisperse liposomes coated with multiple layers (up to 6) as well as cross-linked layersomes were produced. Their coating was firmly confirmed by DLS, zeta potential measurements and by a

'0 FRET experiment, which to date has not yet been employed to demonstrate LbL coating of liposomes. According to the literature, only a limited number of research groups have succeeded in producing layersomes based on biocompatible polymeric species that are stable upon storage, homogeneous, small in size $(<400 \mathrm{~nm})$ and with a large number of layers (>4).(Haidar et al., 2010, 2008; MadrigalCarballo et al., 2010; Ramasamy et al., 2014)

A preliminary evaluation of the developed structures revealed encouraging results, with an increased stability over time especially for 4-layer coated liposomes in the presence of human plasma, 
and for 6-layer coated liposomes in the presence of Triton ${ }^{\mathrm{TM}} \mathrm{X}-100$ and phospholipase $\mathrm{A}_{2}$. This work highlights the importance of the number of layers for the development of more stable liposomes.

10 Although fully interesting results were obtained for multilayered liposomes, the cross-linking of 2layer coated liposomes was somewhat disappointing. The results might suggest that either the reticulation of the coat is incomplete, or it does not have the desired protecting effect.

In any case, this work truly confirms the high potential of LbL coating of liposomes and opens 15 the way to the modification and optimization of liposomes for their pharmaceutical applications. Indeed, the layers wrapped around liposomes could be employed to insert hydrophilic active substances in larger quantities or even prodrugs associated to the polypeptide side chains. Considering the versatility of the LbL approach, this technology could also be applied to other nanoparticles either to stabilize them or to increase their lifespan and therefore provide them a 0 different biodistribution. For instance, it could be used in vaccination for the easy and effective generation of delayed-release forms of nanoparticle-based vaccines.

\section{Acknowledgements}

This work was supported by the Centre National de la Recherche Scientifique (CNRS) and the University of Strasbourg. Florence Hermal was recipient of a 2016-2019 PhD grant from the French Ministère de l'Éducation Nationale, de la Recherche et de la Technologie.

\section{References}

Ai, H., Jones, S.A., Lvov, Y.M., 2003. Biomedical applications of electrostatic layer-by-layer nanoassembly of polymers, enzymes, and nanoparticles. Cell Biochem. Biophys. 39, 23-43. https://doi.org/10.1385/CBB:39:1:23

Blanco, E., Shen, H., Ferrari, M., 2015. Principles of nanoparticle design for overcoming biological 
barriers to drug delivery. Nat. Biotechnol. 33, 941-951. https://doi.org/10.1038/nbt.3330.

Bozzuto, G., Molinari, A., 2015. Liposomes as nanomedical devices. Int. J. Nanomedicine 10, 975-999. https://doi.org/10.2147/IJN.S68861

Bulbake, U., Doppalapudi, S., Kommineni, N., Khan, W., 2017. Liposomal formulations in clinical use: An updated review. Pharmaceutics 9, 12. https://doi.org/10.3390/pharmaceutics9020012 0 Ciobanu, M., Heurtault, B., Schultz, P., Ruhlmann, C., Muller, C.D., Frisch, B., 2007. Layersome: Development and optimization of stable liposomes as drug delivery system. Int. J. Pharm. 344, 154-157. https://doi.org/10.1016/j.ijpharm.2007.05.037

Decher, G., Hong, J.-D., 1991a. Buildup of Ultrathin Multilayer Films by a Self-Assembly Process: I. Consecutive Adsorption of Anionic and Cationic Bipolar Amphiphiles on Charged Surfaces. Makromol. Chem. 46, 321-327. https://doi.org/10.1002/masy.19910460145

Decher, G., Hong, J.-D., 1991b. Buildup of Ultrathin Multilayer Films by a Self-Assembly Process: II. Consecutive Adsorption of Anionic and Cationic Bipolar Amphiphiles and Polyelectrolytes on Charged Surfaces. Berichte der Bunsengesellschaft für Phys. Chemie 95, 1430-1434. https://doi.org/10.1002/bbpc.19910951122

0 Decher, G., Hong, J.-D., Schmitt, J., 1992. Buildup of ultrathin multilayer films by a self-assembly process: III. Consecutively alternating adsorption of anionic and cationic polyelectrolytes on charged surfaces. Thin Solid Films 210-211, 831-835. https://doi.org/10.1016/00406090(92)90417-A

Deng, Z.J., Morton, S.W., Ben-Akiva, E., Dreaden, E.C., Shopsowitz, K.E., Hammond, P.T., 2013. Layer-bylayer nanoparticles for systemic codelivery of an anticancer drug and siRNA for potential triplenegative breast cancer treatment. ACS Nano 7, 9571-9584. https://doi.org/10.1021/nn4047925

Gentile, P., Carmagnola, I., Nardo, T., Chiono, V., 2015. Layer-by-layer Assembly for Biomedical Applications in the Last Decade. Nanotechnology 26, 422001. https://doi.org/10.1088/0957$4484 / 26 / 42 / 422001$

0 Gribova, V., Gauthier-Rouvière, C., Albigès-Rizo, C., Auzely-Velty, R., Picart, C., 2013. Effect of RGD functionalization and stiffness modulation of polyelectrolyte multilayer films on muscle cell 
differentiation. Acta Biomater. 9, 6468-6480. https://doi.org/10.1016/j.actbio.2012.12.015

Guzmán, E., Mateos-Maroto, A., Ruano, M., Ortega, F., Rubio, R.G., 2017. Layer-by-Layer polyelectrolyte assemblies for encapsulation and release of active compounds. Adv. Colloid Interface Sci. 249, 290-307. https://doi.org/doi.org/10.1016/j.cis.2017.04.009

Haidar, Z.S., Hamdy, R.C., Tabrizian, M., 2010. Biocompatibility and safety of a hybrid core-shell nanoparticulate OP-1 delivery system intramuscularly administered in rats. Biomaterials 31, 2746-2754. https://doi.org/10.1016/j.biomaterials.2009.12.034

Haidar, Z.S., Hamdy, R.C., Tabrizian, M., 2008. Protein release kinetics for core-shell hybrid nanoparticles based on the layer-by-layer assembly of alginate and chitosan on liposomes. Biomaterials 29, 1207-1215. https://doi.org/10.1016/j.biomaterials.2007.11.012

Hammond, P.T., 2012. Building biomedical materials layer-by-layer. Mater. Today 15, 196-206. https://doi.org/10.1016/S1369-7021(12)70090-1

Hammond, P.T., 2011. Engineering Materials Layer-by-Layer: Challenges and Opportunities in Multilayer Assembly. AIChE J. 57, 2928-2940. https://doi.org/10.1002/aic.12769

He, H., Lu, Y., Qi, J., Zhu, Q., Chen, Z., Wu, W., 2019. Adapting liposomes for oral drug delivery. Acta Pharm. Sin. B 9, 36-48. https://doi.org/10.1016/j.apsb.2018.06.005

Jacoberger-Foissac, C., Saliba, H., Seguin, C., Brion, A., Kakhi, Z., Frisch, B., Fournel, S., Heurtault, B., 2019. Optimization of peptide-based cancer vaccine compositions, by sequential screening, using '0 versatile liposomal platform. Int. J. Pharm. 562, 342-350. https://doi.org/10.1016/j.ijpharm.2019.03.002

Jain, P., Jain, S., Prasad, K.N., Jain, S.K., Vyas, S.P., 2009. Polyelectrolyte coated multilayered liposomes (Nanocapsules) for the treatment of helicobacter pylori infection. Mol. Pharm. 6, 593-603. https://doi.org/10.1021/mp8002539

5 Jain, S., Kumar, D., Swarnakar, N.K., Thanki, K., 2012. Polyelectrolyte stabilized multilayered liposomes for oral delivery of paclitaxel. Biomaterials 33, 6758-6768. https://doi.org/10.1016/j.biomaterials.2012.05.026

Kakhi, Z., Frisch, B., Bourel-Bonnet, L., Hemmerlé, J., Pons, F., Heurtault, B., 2015. Airway 
administration of a highly versatile peptide-based liposomal construct for local and distant

i0 antitumoral vaccination. Int. J. Pharm. 496, 1047-1056.

https://doi.org/10.1016/j.ijpharm.2015.11.027

Keeney, M., Jiang, X.Y., Yamane, M., Lee, M., Goodman, S., Yang, F., 2015. Nanocoating for biomolecule delivery using layer-by- layer self-assembly. J. Mater. Chem. B 3, 8757-8770. https://doi.org/10.1039/x0xx00000x

5 Kumar, S., Nussinov, R., 2002. Relationship between ion pair geometries and electrostatic strengths in proteins. Biophys. J. 83, 1595-1612. https://doi.org/10.1016/S0006-3495(02)73929-5

Lamichhane, N., Udayakumar, T.S., D’Souza, W.D., Simone II, C.B., Raghavan, S.R., Polf, J., Mahmood, J., 2018. Liposomes: Clinical applications and potential for image-guided drug delivery. Molecules 23, 288. https://doi.org/10.3390/molecules23020288

10 Leguen, E., Chassepot, A., Decher, G., Schaaf, P., Voegel, J.C., Jessel, N., 2007. Bioactive coatings based on polyelectrolyte multilayer architectures functionalized by embedded proteins, peptides or drugs. Biomol. Eng. 24, 33-41. https://doi.org/10.1016/j.bioeng.2006.05.023

Liu, W., Ye, A., Han, F., Han, J., 2019. Advances and challenges in liposome digestion: Surface interaction, biological fate, and GIT modeling. Adv. Colloid Interface Sci. 263, 52-67. https://doi.org/10.1016/j.cis.2018.11.007

Liu, X.Q., Picart, C., 2016. Layer-by-Layer Assemblies for Cancer Treatment and Diagnosis. Adv. Mater. 28, 1295-1301. https://doi.org/10.1002/adma.201502660

Madrigal-Carballo, S., Lim, S., Rodriguez, G., Vila, A.O., Krueger, C.G., Gunasekaran, S., Reed, J.D., 2010. Biopolymer coating of soybean lecithin liposomes via layer-by-layer self-assembly as novel delivery system for ellagic acid. J. Funct. Foods 2, 99-106. https://doi.org/10.1016/j.jff.2010.01.002

Marasini, N., Ghaffar, K.A., Skwarczynski, M., Toth, I., 2017. Liposomes as a Vaccine Delivery System, in: Skwarczynski, Mariusz, Toth, Istvan (Eds.), Micro and Nanotechnology in Vaccine Development. William Andrew Publishing, pp. 221-239. https://doi.org/10.1016/B978-0-323- 
Mladenovska, K., Cruaud, O., Richomme, P., Belamie, E., Raicki, R.S., Venier-Julienne, M.-C., Popovski, E., Benoit, J.P., Goracinova, K., 2007. 5-ASA loaded chitosan-Ca-alginate microparticles: Preparation and physicochemical characterization. Int. J. Pharm. 345, 59-69.

https://doi.org/10.1016/j.ijpharm.2007.05.059

0 Muthu, M.S., Feng, S.-S., 2013. Theranostic liposomes for cancer diagnosis and treatment: current development and pre-clinical success. Expert Opin. Drug Deliv. 10, 151-155. https://doi.org/10.1517/17425247.2013.729576

Nag, O.K., Awasthi, V., 2013. Surface engineering of liposomes for stealth behavior. Pharmaceutics 5, 542-569. https://doi.org/10.3390/pharmaceutics5040542

5 Nguyen, T.X., Huang, L., Gauthier, M., Yang, G., Wang, Q., 2016. Recent advances in liposome surface modification for oral drug delivery. Nanomedicine 11, 1169-1185. https://doi.org/10.2217/nnm.16.9

Petersen, A.L., Hansen, A.E., Gabizon, A., Andresen, T.L., 2012. Liposome imaging agents in personalized medicine. Adv. Drug Deliv. Rev. 64, 1417-1435. https://doi.org/10.1016/j.addr.2012.09.003

Picart, C., Elkaim, R., Richert, L., Audoin, F., Arntz, Y., Cardoso, M.D.S., Schaaf, P., Voegel, J.C., Frisch, B., 2005. Primary cell adhesion on RGD-functionalized and covalently crosslinked thin polyelectrolyte multilayer films. Adv. Funct. Mater. 15, 83-94. https://doi.org/10.1002/adfm.200400106

5 Poon, Z., Lee, J.B., Morton, S.W., Hammond, P.T., 2011. Controlling in Vivo Stability and Biodistribution in Electrostatically Assembled Nanoparticles for Systemic Delivery. Nano Lett. 11, 2096-2103. https://doi.org/10.1021/nl200636r

Ramasamy, T., Haidar, Z.S., Tran, T.H., Choi, J.Y., Jeong, J.-H., Shin, B.S., Choi, H.-G., Yong, C.S., Kim, J.O., 2014. Layer-by-layer assembly of liposomal nanoparticles with PEGylated polyelectrolytes enhances systemic delivery of multiple anticancer drugs. Acta Biomater. 10, 5116-5127. https://doi.org/10.1016/j.actbio.2014.08.021

Richardson, J.J., Björnmalm, M., Caruso, F., 2015. Technology-driven layer-by-layer assembly of 
nanofilms. Science (80-. ). 348, aaa2491. https://doi.org/10.1126/science.aaa2491

Richardson, J.J., Cui, J., Björnmalm, M., Braunger, J.A., Ejima, H., Caruso, F., 2016. Innovation in Layerby-Layer Assembly. Chem. Rev. 116, 14828-14867.

https://doi.org/10.1021/acs.chemrev.6b00627

Rouser, G., Fleischer, S., Yamamoto, A., 1970. Two dimensional thin layer chromatographic separation of polar lipids and determination of phospholipids by phosphorus analysis of spots. Lipids 5, 494-496. https://doi.org/10.1007/BF02531316

.0 Sapsford, K.E., Berti, L., Medintz, I.L., 2006. Materials for fluorescence resonance energy transfer analysis: Beyond traditional donor-acceptor combinations. Angew. Chemie - Int. Ed. 45, 45624588. https://doi.org/10.1002/anie.200503873

Schneider, A., Bolcato-Bellemin, A.L., Francius, G., Jedrzejwska, J., Schaaf, P., Voegel, J.C., Frisch, B., Picart, C., 2006. Glycated polyelectrolyte multilayer films: Differential adhesion of primary vesus tumor cells. Biomacromolecules 7, 2882-2889. https://doi.org/10.1021/bm0605208

Sercombe, L., Veerati, T., Moheimani, F., Wu, S.Y., Sood, A.K., Hua, S., 2015. Advances and challenges of liposome assisted drug delivery. Front. Pharmacol. 6, 286.

https://doi.org/10.3389/fphar.2015.00286

Sun, Y., Rombola, C., Jyothikumar, V., Periasamy, A., 2013. Förster resonance energy transfer microscopy and spectroscopy for localizing protein-protein interactions in living cells. Cytom. Part A 83, 780-793. https://doi.org/10.1002/cyto.a.22321

Tandrup Schmidt, S., Foged, C., Smith Korsholm, K., Rades, T., Christensen, D., 2016. Liposome-based adjuvants for subunit vaccines: Formulation strategies for subunit antigens and immunostimulators. Pharmaceutics 8, 7. https://doi.org/10.3390/pharmaceutics8010007 5 Tryoen-Tóth, P., Vautier, D., Haikel, Y., Voegel, J.C., Schaaf, P., Chluba, J., Ogier, J., 2002. Viability, adhesion, and bone phenotype of osteoblast-like cells on polyelectrolyte multilayer films. J. Biomed. Mater. Res. 60, 657-667. https://doi.org/10.1002/jbm.10110

Vemuri, S., Rhodes, C.T., 1995. Preparation and characterization of liposomes as therapeutic delivery systems: a review. Pharm. Acta Helv. 70, 95-111. https://doi.org/10.1016/0031- 
Weinstein, J.N., Ralston, E., Leserman, L.D., Klausner, R.D., Dragsten, P., Henkart, P., Blumenthal, R., 2018. Self-Quenching Of Carboxyfluorescein Fluorescence: Uses In Studying Liposome Stability And Liposome-Cell Interaction, in: Gregoriadis, G. (Ed.), Liposome Technology: Volume III: Targeted Drug Delivery and Biological Interaction. CRC Press, pp. 183-204. https://doi.org/10.1201/9781351074124

Xing, H., Hwang, K., Lu, Y., 2016. Recent developments of liposomes as nanocarriers for theranostic applications. Theranostics 6, 1336-1352. https://doi.org/10.7150/thno.15464

Zhu, G., Mock, J.N., Aljuffali, I., Cummings, B.S., Arnold, R.D., 2011. Secretory Phospholipase A2 Responsive Liposomes. J. Pharm. Sci. 100, 3146-3159. https://doi.org/10.1002/jps.22530

Fig. 1. Chemical structures of $21 \mathrm{kDa}$ poly(L-lysine) (PLL, $\mathrm{m} \approx 100$, hydrobromide) (A) and of $64 \mathrm{kDa}$ poly(L-glutamic acid) (PGA, $\mathrm{n} \approx 426$, sodium carboxylate) (B) selected for the LbL coating of liposomes.

Fig. 2. Layer-by-layer (LbL) coating procedure of unilamellar liposomes (SUVs, green) with a poly-cationic species (PLL, red) and then with a poly-anionic species (PGA, purple).

Fig. 3. Physico-chemical characterization of 2-layer layersomes. (A) mean diameters and polydispersity indices (PdI); (B) mean zeta potentials. Lp: liposomes; Lp-PLL and Lp-PLL-PGA: layersomes. $\mathrm{n}=5$.

:0 Fig. 4. Size distribution of Lp-PLL-PGA formulations prepared with different concentrations of PLL and PGA. A: Prepared with PLL concentrations ranging from 0.1 to $1.5 \mathrm{mg} \cdot \mathrm{mL}^{-1}$ and PGA concentrations ranging from 0.2 to $3.0 \mathrm{mg} \cdot \mathrm{mL}^{-1}$. B: Prepared with PLL concentrations ranging from 0.1 to $1.5 \mathrm{mg} \cdot \mathrm{mL}^{-1}$ and PGA concentrations ranging from 0.4 to $6.0 \mathrm{mg}$.mL1. $n=3$.

5 Scheme 1. Synthesis of $21 \mathrm{kDa}$ PLL-rhodamine (PLL*) from $21 \mathrm{kDa}$ PLL hydrobromide, rhodamine B isothiocyanate (RITC) and N,N-diisopropylethylamine (DIEA). $\mathrm{n}=\mathrm{x}+\mathrm{y} ;$ when $\mathrm{n} \approx 100, \mathrm{x} \approx 97$ and $\mathrm{y} \approx 3$. 
Scheme 2. Synthesis of 64 kDa PGA-fluorescein (PGA*) from 64 kDa PGA sodium salt, EDC, sulfo-NHS and 5aminofluorescein. $\mathrm{n}=\mathrm{x}+\mathrm{y}$; when $\mathrm{n} \approx 426 ; \mathrm{x} \approx 423 ; \mathrm{y} \approx 3$.

Fig. 5. Emission spectra of fluorescent layersome formulations at the excitation wavelength of fluorescein (488 $\mathrm{nm}$ ). Standard deviations are only shown for fluorescence emission maxima $(\lambda=514 ; 516 \mathrm{~nm}$ and $580 \mathrm{~nm})$. Significantly different fluorescence intensities were observed between 570 and $650 \mathrm{~nm}$ for Lp-PLL*-PGA* formulations excited at 488 $\mathrm{nm}$. Emission spectra were acquired at constant voltage $(500 \mathrm{~V})$ and instrumental correction was performed. $\mathrm{n}=3$ for each formulation.

Fig. 6. Physico-chemical characterization of larger layersomes (A): Mean diameters and polydispersity indices (PdI); (B): Zeta potentials. Lp: liposomes; Lp-PLL and Lp-PLLa $-P_{\mathrm{a}}$ layersomes, with $\mathrm{a}=2$ or 3 and $\mathrm{b}=1,2$ or 3 . $\mathrm{n} \geq 3$.

I0 Fig. 7. Chemical structure of PGA-sulfo-NHS used for the formulation of 2-layer coated and reticulated layersomes (LpPLL-PGA R). $\mathrm{n}=\mathrm{x}+\mathrm{y}$; when $\mathrm{n} \approx 426, \mathrm{x}=\mathrm{y} \approx 213$.

Fig. 8. Percentage of 5(6)-carboxyfluorescein (5(6)-CF) released from 5(6)-CF-liposomes and 5(6)-CF-layersomes in the presence of Triton ${ }^{\mathrm{TM}}$ (A), sPLA $(\mathrm{B})$ and human plasma (C). A: Percentage of 5(6)-CF release with increasing concentrations 15 of Triton ${ }^{\mathrm{TM}} \mathrm{X}-100$. Concentrations of Triton $^{\mathrm{TM}} \mathrm{X}-100$ are expressed as a percentage (w/v). B: Release kinetics of 5(6)-CF from 5(6)-CF-liposomes and 5(6)-CF-layersomes in the presence of sPLA2. $1 \mu \mathrm{L}$ of sPLA2 (0.021 U/mL) was added to $1 \mathrm{~mL}$ suspension (0.008 $\mathrm{mM}$ phospholipids) at $\mathrm{t}=0 \mathrm{~min}$ and $\mathrm{t}=15 \mathrm{~min}$. C: Release kinetics of 5(6)-CF from 5(6)-CF-liposomes and 5(6)-CF-layersomes in the presence of human plasma. Lp: liposomes; Lp-PLLa-PGA and $b=1,2$ or 3; Lp-PLL-PGA R: cross-linked 2-layer layersomes. $\mathrm{n}=3$. 